\title{
A PICARD THEOREM WITH AN APPLICATION TO MINIMAL SURFACES. II
}

\author{
PETER HALL
}

\begin{abstract}
Let $f: \mathbf{C} \rightarrow \mathbf{R}^{n}$ be a parabolic minimal surface such that the normals to $f$ omit $n+k$ directions in general position, $k \geq 0$. We obtain sharp bounds on the dimension of the affine span of $f$ and of the linear span of the Gauss map of $f$.
\end{abstract}

In the first part of this paper [4], we discussed a simply-connected parabolic minimal surface $f: \mathbf{C} \rightarrow \mathbf{R}^{n}$ such that the normals to $f$ omitted $n$ directions in general position. (A minimal surface in what follows will always be a conformal harmonic map.) It was proved that the dimension $d$ of the linear subspace of $\mathbf{C P}^{n-1}$ spanned by the image of the Gauss map of $f$ was at most $n-3$. We now make the stronger assumption that the normals to $f$ omit $n+k$ directions in general position for some positive integer $k$. In Theorem 4 we prove that, if $f$ is nonplanar,

$$
1 \leq d \leq \frac{n-3}{k+1}
$$

If $a$ is the dimension of the affine subspace of $\mathbf{R}^{n}$ spanned by the image of $f$, we prove that

$$
d+3 \leq a \leq \min (n-k d, 2 d+2) .
$$

In Theorem 6 we give examples to show that all values of $d$ and $a$ allowed by the inequalities (1) and (2) occur, for special configurations of $n+k$ directions.

The inequalities (1) and (2) have the curious implication that for given $n$ and $k$ it is not in general possible for the maximum values of $d$ and $a$ to be realised by the same surface. For example, if $f: \mathbf{C} \rightarrow \mathbf{R}^{11}$ is a minimal surface and the normals to $f$ omit 12 directions in general position, then $d \leq 4$, and if $d=4$ then $a \leq 7$. However, there is a minimal surface $f: \mathbf{C} \rightarrow \mathbf{R}^{11}$, the normals to which omit 12 directions in general position, such that $d=3$ and $a=8$.

We remark that, although our methods apply in all cases where the number of omitted normals is at least the number of dimensions of the ambient Euclidean

Received by the editors June 1, 1989.

1980 Mathematics Subject Classification (1985 Revision). Primary 53A10; Secondary 30D35.

Presented to the Third Annual Lehigh University Geometry and Topology Conference, May 28, 1988. 
space, there is another case that appears to be quite different. For a minimal surface $f: \mathbf{C} \rightarrow \mathbf{R}^{4}$, the normals to which omit 3 of the coordinate directions, there may be a restriction on the Gauss map, but if so the reason must be somewhat deep. An equivalent problem has been discussed by M. L. Green [2], to whose paper we refer the interested reader.

After a Euclidean motion, we may suppose that

$$
f: \mathbf{C} \rightarrow \mathbf{R}^{n}=\mathbf{R}^{a} \oplus \mathbf{R}^{n-a}
$$

is a minimal surface such that the projection into $\mathbf{R}^{n-a}$ is constant. The minimal surface $f$ may have holomorphic factors, in the sense that after a Euclidean motion there is an orthogonal decomposition

$$
\mathbf{R}^{a}=\underbrace{\mathbf{R}^{2} \oplus \cdots \oplus \mathbf{R}^{2}}_{h \text { factors }} \oplus \mathbf{R}^{a-2 h}
$$

such the projection of $f$ into each factor is a minimal surface. As Theorem 5 we prove a lower bound on $h$ which extends results of Lawson [5, Proposition 4.3; 6, p. 165, Proposition 16] and Hoffman-Osserman [5, Proposition 4.1]. In Theorem 6 we show that with this restriction all possibilities for $h$ can occur. We remark that, when $a$ is less than $n$, it is sometimes possible to find a larger number of holomorphic factors by considering orthogonal decompositions of the form

$$
\mathbf{R}^{n}=\underbrace{\mathbf{R}^{2} \oplus \cdots \oplus \mathbf{R}^{2}}_{j \text { factors }} \oplus \mathbf{R}^{n-2 j},
$$

which do not respect the subspace spanned by the image of $f$.

The theorems of the first part of this paper are these.

Theorem 1. Let $f: \mathbf{C} \rightarrow \mathbf{R}^{n}$ be a minimal surface and suppose that the normals to $f$ omit $n$ directions in general position. Then $f$ has a holomorphic factor.

Theorem 2. Let

$$
\Phi: \mathbf{C} \rightarrow Q_{n-2} \subset \mathbf{C} \mathbf{P}^{n-1}
$$

be holomorphic. Suppose that $\Phi$ does not meet the union of $n$ hyperplanes $\Pi_{1}, \ldots, \Pi_{n}$ with real coefficients and in general position. Then the image of $\Phi$ lies in a linear subspace of codimension 2 , and hence in a hyperplane tangent to $Q_{n-2}$.

Theorem 1 was obtained by translating the conditions on the minimal surface into conditions on its Gauss map and applying Theorem 2. The same translation will yield Theorem 4 below, but rather than explicitly proving a generalization of Theorem 2, we apply Theorem 2 together with Theorem 3, which is a Picard theorem due to M. L. Green.

Theorem 3 (Green [1, Theorem 2]). Let $h: \mathbf{C} \rightarrow \mathbf{C P}^{m}$ be a holomorphic curve that omits $m+2$ distinct hyperplanes. Then the image of $h$ lies in some hyperplane of $\mathbf{C P}^{m}$. 
Theorem 4. Let $f: \mathbf{C} \rightarrow \mathbf{R}^{n}$ be a nonconstant minimal surface and suppose that the normals to $f$ omit $n+k$ directions in general position, where $k \geq 0$. Let

$$
\begin{aligned}
d= & \text { dimension of linear subspace of } \mathbf{C P}^{n-1} \\
& \text { spanned by Gauss map of } f, \\
a= & \text { dimension of affine subspace of } \mathbf{R}^{n} \\
& \text { spanned by } f .
\end{aligned}
$$

Then

$$
\begin{aligned}
& 1 \leq d \leq \frac{n-3}{k+1}, \\
& d+3 \leq a \leq \min (n-k d, 2 d+2) .
\end{aligned}
$$

Proof. Without loss of generality, the affine span of the minimal surface $f$ is a linear subspace $\mathbf{R}^{a}$ of $\mathbf{R}^{n}$, and we use the orthogonal decomposition

$$
\mathbf{R}^{n}=\mathbf{R}^{a} \oplus \mathbf{R}^{n-a} \text {. }
$$

Let the given vectors omitted by the normals to $f$ be $v_{1}, \ldots, v_{n+k}$. Each $v_{i}$ may be written as $v_{i}=u_{i}+w_{i}$, with $u_{i} \in \mathbf{R}^{a}$ and $w_{i} \in \mathbf{R}^{n-a}$. We remark that $u_{i} \neq 0$ since $w_{i}$ is always normal to $f$.

Let $\Phi: \mathbf{C} \rightarrow \mathbf{C P}^{n-1}$ be the Gauss map of $f$, regarded as a holomorphic curve. We have the inclusions

$$
\Phi(\mathbf{C}) \subset \mathbf{C} \mathbf{P}^{d} \subset \mathbf{C P}^{a-1} \subset \mathbf{C P}^{n-1} .
$$

Here $\mathbf{C P} \mathbf{P}^{d}$ is the span of the Gauss map of $f ; \mathbf{C P}^{a-1}$ is the subspace of $\mathbf{C P}^{n-1}$ corresponding to $\mathbf{R}^{a}$ in (4).

Let $\pi: \mathbf{R}^{n} \rightarrow \mathbf{R}^{a}$ be projection on to the first factor of (4); then $\pi \circ f$ is a minimal surface in $\mathbf{R}^{a}$. Since the $v_{i}$ span $\mathbf{R}^{n}$, the $u_{i}$ span $\mathbf{R}^{a}$. If $\left(u_{1}, \ldots, u_{a}\right)$ is a basis for $\mathbf{R}^{a}$, it consists of $a$ directions in general position omitted by the normals to $\pi \circ f$, and Theorem 2 implies that

$$
d-3 \leq a \text {. }
$$

Each of the vectors $u_{i}, i=1, \ldots, n+k$, corresponds to a hyperplane $\Pi_{i}$ of $\mathbf{C P}^{a-1}$, and $\Pi_{i}$ meets $\mathbf{C P} \mathbf{P}^{d}$ in a hyperplane of $\mathbf{C P}^{d}$. Since the $u_{i} \operatorname{span} \mathbf{R}^{a}$, there are $d+1$ such hyperplanes $\Lambda_{1}, \ldots, \Lambda_{d+1}$ in general position in $\mathbf{C P}^{d}$. Now $\Phi$ is not contained in any linear subspace of $\mathbf{C P}^{d}$, so that by Theorem 3 there cannot be $d+2$ distinct hyperplanes of $\mathbf{C P}^{d}$ that are omitted by $\Phi$. Therefore each of $\Pi_{1}, \ldots, \Pi_{n+k}$ must pass through some one of $\Lambda_{1}, \ldots, \Lambda_{d+1}$.

Partition the vectors $u_{i}, i=1, \ldots, n+k$, into $d+1$ subsets corresponding to hyperplanes $\Pi_{i}$ which pass through $\Lambda_{1}, \ldots, \Lambda_{d+1}$. Each subset spans a subspace of $\mathbf{R}^{a}$ of positive dimension and the subspaces corresponding to distinct $\Lambda_{j}$ intersect only at the origin. Thus we obtain a decomposition of $\mathbf{R}^{a}$ as the direct sum of $d+1$ subspaces, not in general orthogonal. 
We claim that each subspace in the decomposition has dimension 1 or 2 . Consider any of the $\Lambda_{j}$, and let $\rho: \mathbf{R}^{n} \rightarrow \mathbf{R}^{p}$ be orthogonal projection on to the corresponding subspace. In the standard coordinate system on $\mathbf{R}^{n}$, the Gauss map $\Phi$ of $f$ is defined in this manner. Let

$$
\varphi_{m}=\frac{\partial f_{m}}{\partial x}-i \frac{\partial f_{m}}{\partial y}, \quad m=1, \ldots, n,
$$

obtaining a holomorphic map $\varphi: \mathbf{C} \rightarrow \mathbf{C}^{n}$. The Gauss map $\Phi: \mathbf{C} \rightarrow \mathbf{C P}^{n-1}$ is given in homogeneous coordinates by (6), treating the zeros of $\varphi$ as removable singularities. To recover the component of $f$ in the direction of a unit vector $v \in \mathbf{R}^{n}$, we form $v \cdot \varphi$, integrate and take the real part. Now, if $v$ lies in $\mathbf{R}^{p}$ corresponding to $\Lambda_{j}, v \cdot \varphi$ is some complex multiple of the value on $\varphi$ of the linear form defining $\Lambda_{j}$. Hence every direction $v \in \mathbf{R}^{p}$ yields a coordinate function which is some linear combination of the real and imaginary parts of the same integral. Since $\rho \circ f$ is full in $\mathbf{R}^{d}, p$ is 1 or 2 .

We have constructed a direct decomposition of $\mathbf{R}^{a}$, not in general orthogonal, into copies of $\mathbf{R}^{2}$ and $\mathbf{R}$. Since the number of factors is $d+1$, there must be $a-d-1$ copies of $\mathbf{R}^{2}$ and $2 d-a+2$ copies of $\mathbf{R}$ :

$$
\mathbf{R}^{a}=\underbrace{\mathbf{R}^{2} \oplus \cdots \oplus \mathbf{R}^{2}}_{a-d-1 \text { factors }} \oplus \underbrace{\mathbf{R} \oplus \cdots \oplus \mathbf{R}}_{2 d-a+2 \text { factors }} .
$$

This shows in particular that

$$
a \leq 2 d+2 \text {. }
$$

Each of $u_{1}, \ldots, u_{n+k}$ must lie in one of the factors in (7). General position means that any $n$ of the vectors $v_{1}, \ldots, v_{n+k} \operatorname{span} \mathbf{R}^{n}$. Therefore any $n$ of $u_{1}, \ldots, u_{n+k}$ must $\operatorname{span} \mathbf{R}^{a}$. It follows that at least $k+1$ of the $u_{i}$ must lie in each copy of $\mathbf{R}$ and at least $k+2$ of the $u_{i}$ must lie in each copy of $\mathbf{R}^{2}$. This yields the inequality

$$
\begin{aligned}
(k+1) & \times \text { number of copies of } \mathbf{R} \\
& +(k+2) \times \text { number of copies of } \mathbf{R}^{2} \\
= & (k+1)(2 d-a+2)+(k+2)(a-d-1) \\
= & k d+k+a \\
\leq & n+k,
\end{aligned}
$$

or equivalently

$$
a \leq n-k d
$$

Combining (5) and (9) we obtain

$$
d+3 \leq a \leq n-k d
$$

so that

$$
d \leq \frac{n-3}{k+1}
$$


Since $f$ is nonplanar, we have also

$$
1 \leq d
$$

The inequalities (5), (8), (9), (10) and (11) establish the assertions (1) and (2) of the theorem.

Theorem 5. Let $F$ be a Riemann surface and let $f: F \rightarrow \mathbf{R}^{n}$ be minimal. Let $\Phi: F \rightarrow Q_{n-2} \subset \mathbf{C P}^{n-1}$ be the Gauss map of $f$. The image of $\Phi$ lies in the polar with respect to $Q_{n-2}$ of an $(h-1)$-dimensional linear subspace of $Q_{n-2}$ if and only if there is an orthogonal decomposition

$$
\mathbf{R}^{n}=\underbrace{\mathbf{R}^{2} \oplus \cdots \oplus \mathbf{R}^{2}}_{h \text { factors }} \oplus \mathbf{R}^{n-2 h},
$$

such that the projection of $f$ into each factor is a minimal surface. In particular, if the dimension $d$ of the linear span of $\Phi$ is less than or equal to $n-2 h-1$, then the decomposition (12) exists.

Remark. If $n=2 h+2$, Theorem 5 yields the theorem of Lawson [5, Proposition $4.3 ; 6$, p. 165 , Proposition 16] that a minimal surface is a holomorphic curve in some orthogonal complex structure if and only if its Gauss map lies in a linear subspace of $Q_{n-2}$. The polar of a point of $Q_{n-2}$ is the tangent space at that point, so that, if $h=1$, Theorem 5 yields the theorem of Hoffman and Osserman [5, Proposition 4.1] that $f$ has a holomorphic factor if and only if its Gauss map lies in a hyperplane tangent to $Q_{n-2}$. Our argument is modelled on that of Hoffman and Osserman.

Proof. Suppose that $\Lambda$ is an $(h-1)$-dimensional linear subspace of $Q_{n-2}$ and that $\Phi(F) \subset \operatorname{polar}(\Lambda)$. Let $p_{1}, \ldots, p_{n}$ be points of $\Lambda$ such that each of them is polar to the others. Each of the $p_{j}, j=1, \ldots, h$, can be written in homogeneous coordinates as $p_{j}=\left(\alpha_{j}+i \beta_{j}\right)$, where $\alpha_{j}$ and $\beta_{j}$ are a pair of real unit vectors. The two-dimensional subspaces of $\mathbf{R}^{n}$ spanned by the pairs $\left(\alpha_{j}, \beta_{j}\right)$ give rise to the orthogonal decomposition (12). For $j=1, \ldots, h$ we have the relation $p_{j} \cdot \Phi(z)=0$ for all $z \in F$, expressing the fact that

$$
\Phi(F) \subset \operatorname{polar}(\Lambda) \subset \operatorname{polar}\left(\left\{p_{j}\right\}\right) .
$$

This implies that the projection of $f$ into the corresponding $\mathbf{R}^{2}$ factor of (12) is conformal or anticonformal. To prove the converse, suppose that there exists an orthogonal decomposition of the form (12) such that the projection into each factor is a minimal surface. Let $\left(\alpha_{j}, \beta_{j}\right), j=1, \ldots, h$, be an orthogonal basis for each $\mathbf{R}^{2}$ factor. Then the points $p_{j}=\left(\alpha_{j}+i \beta_{j}\right)$ span an $(h-1)$-dimensional linear subspace $\Lambda$ of $Q_{n-2}$ and $\Phi(F) \subset \operatorname{polar}(\Lambda)$.

To prove the last assertion, suppose that $L \subset \mathbf{C} \mathbf{P}^{n-1}$ is a linear subspace of dimension $n-2 h-1$ containing $\Phi(F)$. Then $\operatorname{polar}(L) \subset \mathbf{C P}^{n-1}$ is a linear subspace of dimension $2 h$. The intersection of $Q_{n-2}$ with $\operatorname{polar}(L)$ is 
a quadric variety of dimension $2 h-1$, hence contains a linear subspace of dimension $h-1$ (see for example [3, p. 735]). We have

$$
\Lambda \subset \operatorname{polar}(L)
$$

and hence

$$
\Phi(F) \subset L \subset \operatorname{polar}(\Lambda)
$$

We conclude by constructing examples to show that the results of Theorems 4 and 5 are sharp.

Theorem 6. Let $n, k, d$, and a satisfy the inequalities (1) and (2) of Theorem 4. Then there exists a minimal surface $f: \mathbf{C} \rightarrow \mathbf{R}^{n}$ such that the normals to $f$ omit $n=k$ directions in general position and $f$ has the given values of $d$ and $a$. If $\frac{1}{2}(a-d-2) \leq h \leq \frac{1}{2}(a-3)$ or $a=2 h$, then $f$ can be constructed so that $h$ is the number of $\mathbf{R}^{2}$ factors in the orthogonal decomposition

$$
\mathbf{R}^{a}=\mathbf{R}^{2} \oplus \cdots \oplus \mathbf{R}^{2} \oplus \mathbf{R}^{a-2 h} .
$$

Proof. First consider the case $a=n$, which occurs only when $k=0$. The value of $d$ may be any integer satisfying

$$
\frac{1}{2}(n-2) \leq d \leq n-3
$$

For $d=n-3$ we give a construction from Remark 1 at the end of Part I of this paper [4]. If $\left(e_{1}, \ldots, e_{n}\right)$ is the standard orthonormal basis for $\mathbf{R}^{n}$, the $n$ lacunary normals may be taken to be

$$
\begin{gathered}
v_{1}=e_{1}, \quad v_{2}=e_{2}, \\
v_{3}=\frac{1}{\sqrt{2}}\left(e_{1}+e_{3}\right), \quad v_{4}=e_{4}, \\
v_{5}=\frac{1}{\sqrt{2}}\left(e_{1}+e_{5}\right), \quad v_{6}=\frac{1}{\sqrt{2}}\left(e_{1}+e_{6}\right), \\
\vdots \\
v_{n-1}=\frac{1}{\sqrt{2}}\left(e_{1}+e_{n-1}\right), \quad v_{n}=e_{n} .
\end{gathered}
$$

If $\left(g_{1}, \ldots, g_{n}\right)$ are homogeneous coordinates of the Gauss map in the system of coordinates dual to $\left(v_{1}, \ldots, v_{n}\right)$, we may choose

$$
\begin{aligned}
& \sqrt{n-3} g_{1}=i g_{2}=e^{z}, \\
& \sqrt{2} g_{3}=i g_{4}=e^{2 z}, \\
& g_{5}^{2}=\sqrt{2} g_{1} g_{3}, \\
& g_{6}^{2}=\sqrt{2} g_{1} g_{5}, \\
& \quad \vdots \\
& g_{n}^{2}=\sqrt{2} g_{1} g_{n-1} .
\end{aligned}
$$


The formulas (15) may be integrated to obtain an immersed minimal surface with Gauss map (15).

To obtain examples with $d=n-j-3, j \geq 1$, we modify the set of vectors (14) to obtain:

$$
\begin{aligned}
& v_{1}=e_{1}, \quad v_{2}=e_{2}, \\
& v_{3}=\frac{1}{\sqrt{2}}\left(e_{1}+e_{3}\right), \quad v_{4}=e_{4}, \\
& v_{5}=\frac{1}{\sqrt{2}}\left(e_{1}+e_{5}\right), \quad v_{6}=e_{6}, \\
& \quad \vdots \\
& v_{2 j+3}=\frac{1}{\sqrt{2}}\left(e_{1}+e_{2 j+3}\right), \quad v_{2 j+4}=e_{2 j+4}, \\
& v_{2 j+5}=\frac{1}{\sqrt{2}}\left(e_{1}+e_{2 j+5}\right), \quad v_{2 j+6}=\frac{1}{\sqrt{2}}\left(e_{1}+e_{2 j+6}\right), \\
& \vdots \quad v_{n}=e_{n} .
\end{aligned}
$$

A corresponding modification of the components of the Gauss map (15) gives:

$$
\begin{aligned}
& \sqrt{n-j-3} g_{1}=i g_{2}=e^{z}, \\
& \sqrt{2} g_{3}=i g_{4}=e^{2 z}, \\
& g_{5}^{2}=-2 g_{6}^{2}=2 \sqrt{2} g_{1} g_{3}, \\
& \quad \quad \vdots \\
& g_{2 j+3}^{2}=-2 g_{2 j+4}^{2}=2 \sqrt{2} g_{1} g_{2 j+1}, \\
& g_{2 j+5}^{2}=\sqrt{2} g_{1} g_{2 j+3}, \\
& \quad \vdots \\
& g_{n}^{2}=\sqrt{2} g_{1} g_{n-1} .
\end{aligned}
$$

For any of the examples described by (14), (15) or (16), (17), the polar with respect to $Q_{n-2}$ of the linear span $\mathbf{C P}^{d}$ of the Gauss map is an $(n-d-2)$ dimensional linear space $L \subset \mathbf{C P}^{n-1}$. It may be computed that the linear space $L$ intersects $Q_{n-2}$ in a quadric $Q_{n-d-3}$ which is nonsingular. Therefore the dimension of a maximal linear subspace of $Q_{n-d-3}$ has the smallest possible value, namely $\frac{1}{2}(n-d-3)$ if $n-d$ is odd and $\frac{1}{2}(n-d-4)$ if $n-d$ is even [3, p. 735]. This implies that, in the decomposition (3), the examples (14), (15) and (16), (17) have the smallest number of holomorphic factors permitted by Theorem 5. 
At the other extreme, an example with $a=n$ and the largest possible number of holomorphic factors is the holomorphic curve $f: \mathbf{C} \rightarrow \mathbf{C}^{m}$ given by

$$
f(z)=\left(e^{3 z}, e^{4 z}, \ldots, e^{(m+2) z}\right),
$$

considered as a minimal surface in $\mathbf{R}^{2 m}$, the normals to which omit the coordinate directions. To obtain any intermediate number of holomorphic factors we take the Cartesian product of examples of the types (15) or (17) and (18).

We now construct examples $a<n$ and $k$ not necessarily zero. Our construction is based on the proof of Theorem 4. Take a full minimal surface in $\mathbf{R}^{a}$ which is any of those just constructed. Corresponding to the orthogonal decomposition

$$
\mathbf{R}^{n}=\mathbf{R}^{a} \oplus \mathbf{R}^{n-a},
$$

each lacunary normal must be of the form $v_{i}=u_{i}+w_{i}$, with $0 \neq u_{i} \in \mathbf{R}^{a}$ and $w_{i} \in \mathbf{R}^{n-a}$. Take $w_{1}, \ldots, w_{n+k}$ to be any vectors in general position in $\mathbf{R}^{n-a}$. We have seen in the proof of Theorem 4 that there is a direct decomposition

$$
\mathbf{R}^{a}=\underbrace{\mathbf{R}^{2} \oplus \cdots \oplus \mathbf{R}^{2}}_{a-d-1 \text { factors }} \oplus \underbrace{\mathbf{R} \oplus \cdots \oplus \mathbf{R}}_{2 d-a-2 \text { factors }}
$$

and each of the $u_{i}$ must lie in one of the factors. The $v_{i}$ are in general position provided there are at least $k+1$ of the $u_{i}$ in each $\mathbf{R}$ factor and at least $k+2$ of the $u_{i}$ in each $\mathbf{R}^{2}$ factor. That can be achieved provided the inequalities (1) and (2) in the conclusion of Theorem 4 are satisfied.

\section{ACKNOWLEDGMENT}

I am grateful to M. L. Green and H. B. Lawson, Jr., for their help and encouragement.

\section{REFERENCES}

1. M. L. Green, Holomorphic maps into complex projective spaces omitting hyperplanes, Trans. Amer. Math. Soc. 169 (1972), 89-103.

2. M. L. Green, On the functional equation $f^{2}=e^{2 \varphi_{1}}+e^{2 \varphi_{2}}+e^{2 \varphi_{3}}$ and a new Picard theorem, Trans. Amer. Math. Soc. 195 (1974), 223-230.

3. P. Griffiths and J. Harris, Principles of algebraic geometry, Wiley, New York, 1978.

4. P. Hall, A Picard theorem with an application to minimal surfaces, Trans. Amer. Math. Soc. . 314 (1989), 597-603.

5. D. A. Hoffman and R. Osserman, The geometry of the generalized Gauss map, Mem. Amer. Math. Soc., vol. 28, no. 236, 1980.

6. H. B. Lawson, Jr., Lectures on minimal submanifolds. I, Publish or Perish, Berkeley, Calif., 1980.

Department of Mathematics, Washington University, St. Louis, Missouri 63130

Current address: Department of Mathematics, The University of Alabama, P.O.B. 870350, Tuscaloosa, Alabama 35487-0350 